\title{
Lost in Translation: Regulation of ABCG2 Expression in Human Embryonic Stem Cells
}

\section{Raji Padmanabhan ${ }^{1}$, Kevin G Chen ${ }^{2}$ and Michael M Gottesman ${ }^{1 *}$}

${ }^{1}$ Laboratory of Cell Biology, National Cancer Institute, National Institutes of Health, Bethesda, MD 20892, USA

${ }^{2}$ NIH Stem Cell Unit, National Institute of Neurological Disorders and Stroke, National Institutes of Health, Bethesda, MD 20892, USA

\begin{abstract}
The expression and function of the ATP-binding cassette (ABC) transporter ABCG2 have been studied for two decades in both adult and cancer stem cells. However, this important $A B C$ transporter has not been well characterized in human embryonic stem cells (hESCs). Studies designed to understand the role of ABCG2 in hESCs are still in their initial stages. Several recent reports on expression patterns of the ABCG2 gene in $\mathrm{hESCs}$ contain contradictory results at both the mRNA and protein levels. In this review, we provide possible explanations for these discrepancies in ABCG2 expression patterns. We discuss micro-RNA-mediated regulatory roles in controlling ABCG2 mRNA stability and translation, which are associated with hESC pluripotency and differentiation.
\end{abstract}

Keywords: Human embryonic stem cells; Pluripotency; Differentiation; ATP-binding cassette; ABCG2

\section{Introduction}

ABCG2, a 72-kDa half transporter belonging to the ATP Binding Cassette $(\mathrm{ABC})$ super family of proteins, forms a functional homodimer or oligomer that actively effluxes structurally unrelated metabolites and xenobiotics from mammalian cells [1-3]. Normally expressed in the gastrointestinal tract, kidney, liver, blood-testis, blood-brain, and maternal fetal barriers, ABCG2 is thought to serve a protective role by pumping out toxic compounds [1-3]. However, its expression in several types of solid and hematological cancers may result in multidrug resistance (MDR) and may also serve as a marker for cancer prognosis [2]. In addition, ABCG2, implicated as a marker for stem cells through its ability to pump out Hoechst 33342 dye, creates a side population (SP), which has been used to identify neural, mesodermal, hematopoietic stem/progenitor cells, and several adult and cancer stem cell populations [1-8]. Despite ABCG2's possible roles as a cell protector, a mediator of MDR, and a stem cell marker, questions remain to be addressed about the true physiological function of ABCG2, including its role in self-renewal and pluripotency. Human embryonic stem cells (hESCs), characterized by self-renewal and pluripotency, may provide some insights into fundamental biological processes and disease modeling, thus opening the door to many important applications such as regenerative medicine and pharmaceutical development. Therefore, it is imperative to understand the roles of ABCG2 in mediating hESC pluripotency, cellular homeostasis, and cell fate commitments. We should point out that although hESC growth in vitro provides a functional cell resource for regenerative medicine, it remains unclear whether differentiated cells from hESCs in vitro are physiologically relevant when compared with mammalian cells in vivo. With regard to this issue, we should be aware of the possibility that hESCs cultured in vitro may not completely reflect the physiological status of ABCG2 in vivo.

To understand the role of ABCG2 in the regulation of hESCs, three different groups studied the expression of ABCG2 in different hESC lines. Zeng et al. [5] found that both the ABCG2 mRNA and protein were undetectable in three hESC lines (i.e., H9, HUES1, and CT2). In contrast, Apati et al. [6] reported that both the ABCG2 mRNA and protein were expressed in HUES1 and HUES9 cells. To resolve the controversy, we carried out similar studies in a panel of hESC lines that have been extensively characterized in the NIH
Stem Cell Unit. We found that ABCG2 mRNA was expressed in all examined hESC lines including WA01, WA09, BG01, and BG03 [4]. Interestingly, ABCG2 protein expression could not be detected by Western blot and immunofluorescence microscopy regardless of the presence of intermediate levels of ABCG2 mRNAs in these cells [4]. The specificities of PCR primers and antibodies have been extensively validated in different cell types. For Western blotting, the anti-ABCG2 (BXP21) monoclonal antibody provides conclusive results, whereas the monoclonal antibody 5D3 is able to localize the ABCG2 protein on the plasma membrane by immunofluorescent staining and flow cytometric analysis $[4,6]$.

These inconsistent results might stem from a variety of issues. First, the apparent absence of $A B C G 2$ mRNA expression (as reported by Zeng et al. [5]) may be due to a lack of sensitivity in their detection methods. With a highly sensitive method (e.g., quantitative real-time PCR), we have confirmed $A B C G 2 \mathrm{mRNA}$ expression. Second, different forms of cellular stress and differentiation signals regulate ABCG2 expression, which may be the reason for differences in the protein expression profiles among different hESC lines [4-8]. In general, contradictory results in hESC research can be due to different cellular states, suboptimal growth conditions, and differential handling of hESC culture in vitro. In particular, hESC experiments could be greatly influenced by growth media containing various growth factors, extracellular matrices, environmental cues, and various growth patterns [9]. Suboptimal growth factors may definitely result in alterations of core signaling pathways, which underlie epithelial-to-mesenchymal transitions (EMTs), cellular heterogeneity, and chromosomal instability in hESCs [10]. All the above conditions would alter ABCG2 expression and localization.

*Corresponding author: Michael M. Gottesman, Laboratory of Cell Biology, National Cancer Institute, National Institutes of Health, Bethesda, MD 20892, USA Tel: 301496 1530; Fax: 301402 0450; E-mail: mgottesman@nih.gov

Received February 12, 2014; Accepted March 15, 2014; Published March 17 2014

Citation: Padmanabhan R, Chen KG, Gottesman MM (2014) Lost in Translation: Regulation of ABCG2 Expression in Human Embryonic Stem Cells. J Stem Cell Res Ther 4: 180. doi:10.4172/2157-7633.1000180

Copyright: (c) 2014 Padmanabhan R, et al. This is an open-access article distributed under the terms of the Creative Commons Attribution License, which permits unrestricted use, distribution, and reproduction in any medium, provided the original author and source are credited. 
Citation: Padmanabhan R, Chen KG, Gottesman MM (2014) Lost in Translation: Regulation of ABCG2 Expression in Human Embryonic Stem Cells. J Stem Cell Res Ther 4: 180. doi:10.4172/2157-7633.1000180

Alternatively, the differences in ABCG2 expression may result from altered gene expression profiles that change the regulation of $A B C G 2$ at the transcriptional, post-transcriptional, translational, and/ or post translational levels. Changes in transcription of $A B C G 2$ could arise from differences in epigenetic modifications, transcription factor expression or function, and alternative promoter usage (Figure 1A).
A number of studies have been published on $A B C G 2$ transcription in cancer samples, cancer cell lines, and stem cells, which have been reviewed elsewhere $[1-3,11]$. Little is known about the regulation of ABCG2 in hESCs.

One possible mechanism by which $A B C G 2$ expression in undifferentiated hESCs is regulated transcriptionally is via pre-mRNA

A

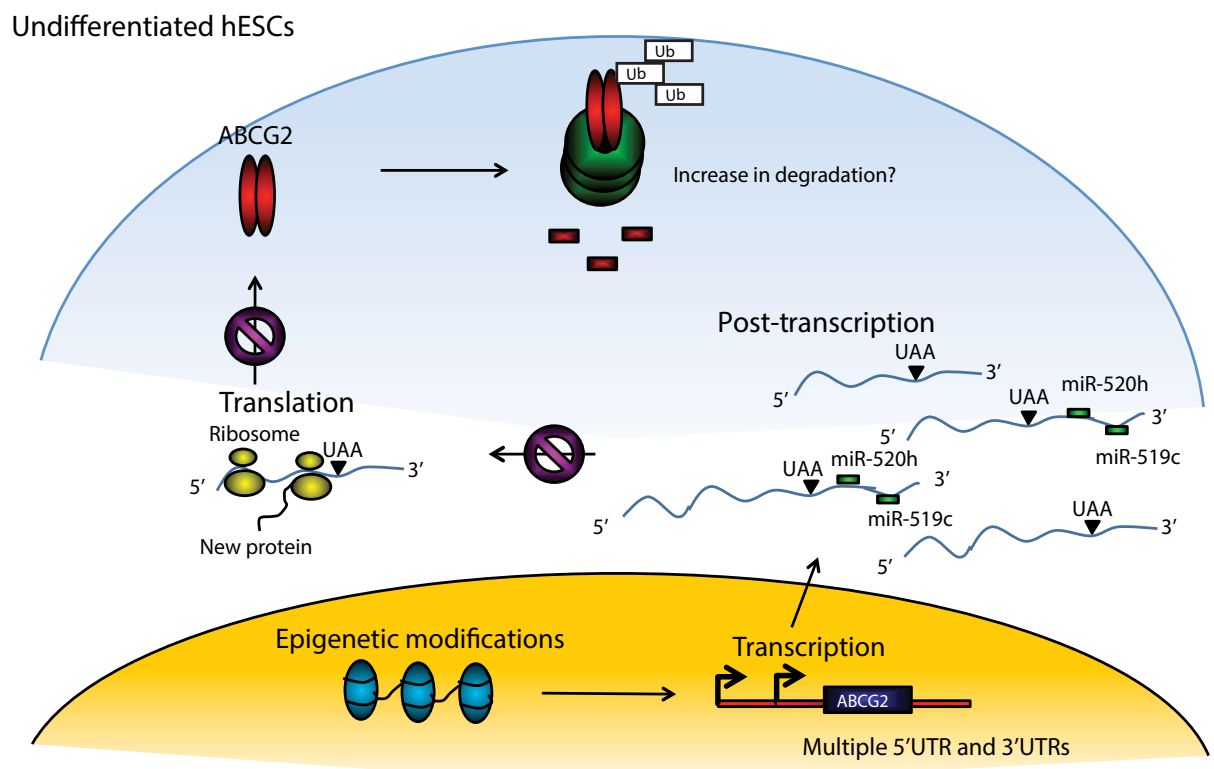

B

Differentiation, hypoxia/reperfusion, physical or UV-induced stress of hESCs

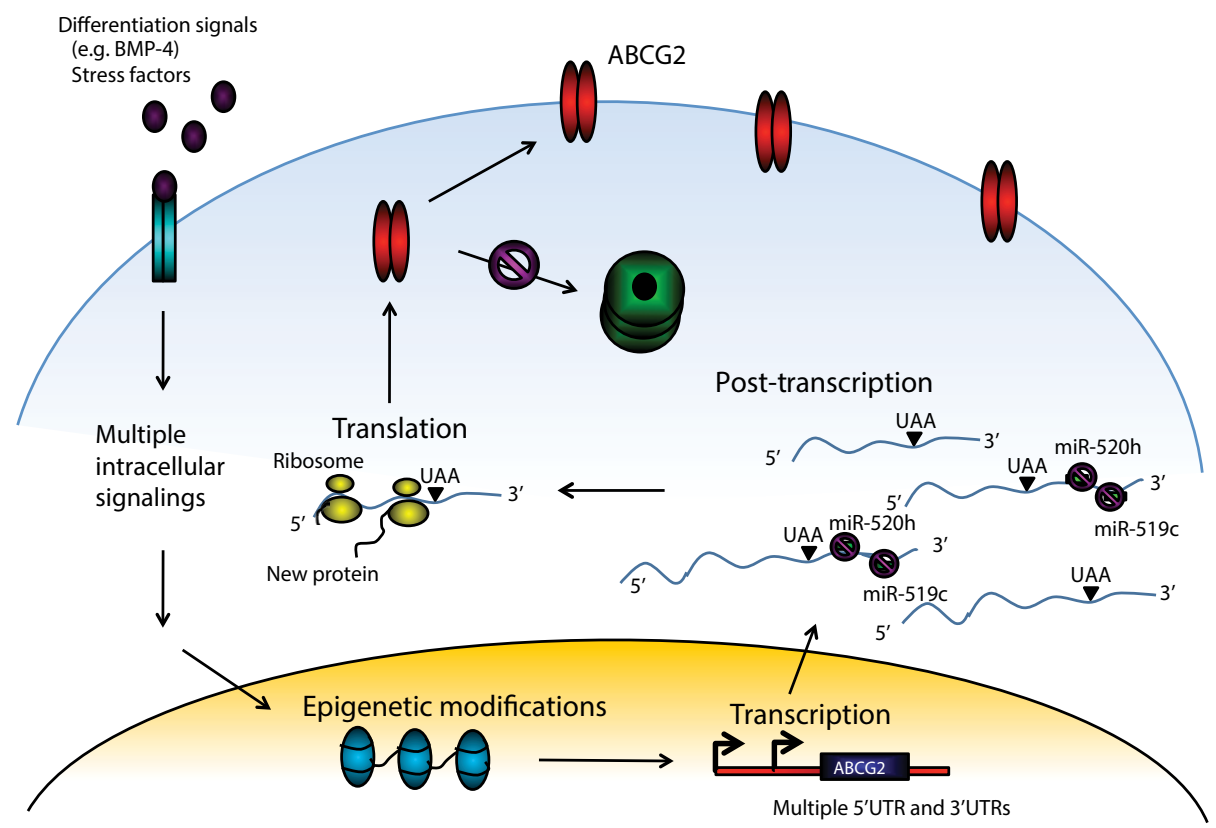

Figure 1: Regulation of $A B C G 2$ in undifferentiated or differentiated, hypoxia/reperfusion treated, physical or UV induced stress treated hESCs. (A) Undifferentiated hESCs express $A B C G 2$ mRNA but lack ABCG2 protein. Whereas $A B C G 2$ is down regulated by miR-519c and miR-520h, alternative mechanisms for loss of ABCG2 protein expression are alternative expression of 5' and 3' UTRs, repression of translation, and increased proteasome-mediated degradation. (B) Upon differentiation, hypoxia/reperfusion, physical or UV-induced stress, hESCs have altered regulation of $A B C G 2$ allowing protein to be expressed. Mechanisms might include the use of alternative 5' and $3^{\prime}$ UTRs, decreased expression of miRNAs, increased translation, and decreased protein degradation. 
processing. Alternative splicing of pre-mRNA has been involved in some $A B C$ transporter genes. We previously determined that the $A B C B 5$ locus generates fragments of $A B C B 5$ of various lengths, which might be due to alternative splicing of the $A B C B 5$ pre-mRNA [12]. The functional implications of various $A B C B 5$ mRNA products remain to be determined. However, one interesting report showed that alternative pre-mRNA splicing has been linked to missense mutations and single nucleotide polymorphisms (SNPs) of the $A B C B 11$ gene and implicated in intrahepatic cholestasis due to bile salt export deficiency [13]. So far, no $A B C G 2$ pre-mRNA regulation studies have been reported in hESCs. It would be interesting to verify this possible regulation in future studies by using $A B C G 2$ intron-specific real-time PCR in hESCs under various growth conditions.

Another possible mechanism by which ABCG2 expression in undifferentiated hESCs is regulated post-transcriptionally is through alternative usage of the two 3'UTRs (Figure 1A). Two possible polyadenylation sites in $A B C G 2$ mRNA result in a short and long 3' UTR $[6,14,15]$. The hESC lines HUES9 and HUES1 only express ABCG2 with a short 3' UTR [6]. The shorter 3' UTR lacks putative microRNA (miRNA) binding sites [14,15], which could result in increased mRNA stability and ultimately lead to increased ABCG2 protein expression, as previously reported $[6,14,15]$. Post transcriptional regulation of $A B C G 2$ can be achieved through miRNA interference (Figure 1A). Expression of the two miRNAs, miR-519c and miR-520h, is inversely correlated with ABCG2 protein levels in hESC lines WA09 and WA01 [4]. BMP4-mediated differentiation caused a 9.2-fold reduction in both miR519c and miR-520h in WA01 hESCs after 144 hours. However, with undifferentiated cells, only a 2 -fold reduction in the same miRNAs was shown under the same conditions in WA09 cells [4]. Decreased expression of the two miRNAs corresponded to an increase in ABCG2 protein expression [4]. Furthermore, transfection of the two inhibitors of miR-519c and miR-520h into BMP4-treated WA09 and WA01 cells modulated ABCG2 protein expression; whereas introduction of the corresponding mimics of miR-519c and miR-520h decreased ABCG2 protein expression in the WA09 cells [4]. A number of other miRNAs may be involved in regulating ABCG2 mRNA expression. Future studies should determine whether ABCG2 protein expression in hESCs might result from altered miRNA expression and differential utilizations of the 3'UTRs of the ABCG2 gene.

Posttranslational modifications, localization, and protein interactions could also alter ABCG2 expression and function (Figure 1A). Functional ABCG2 protein may require N-glycosylation and Pim1 -mediated phosphorylation [16,17]. Furthermore, a functional PI3K/ Akt pathway has been implicated in ABCG2 localization and functional expression in stem cells $[18,19]$. Future studies should determine: (i) whether post translational regulation of ABCG2 through signaling is cell- and lineage-type-dependent and (ii) the roles of key signaling pathways in the regulation of ABCG2 protein stability, localization and function.

Two prominent microenvironmental mechanisms by which ABCG2 is regulated are differentiation and hypoxia (Figure 1B). As shown in our study, only BMP-4-mediated differentiation, not spontaneous or embryoid body differentiation, resulted in ABCG2 protein expression [4]. Post-hypoxia/reoxygenation in the hESC lines BG01 and $\mathrm{H} 9$ resulted in the emergence of an $\mathrm{ABCG}^{+} / \mathrm{SSEA3}^{+}$ population in undifferentiated hESCs, which were characterized by low p53 expression and high HIF-2alpha, NANOG, and OCT4 expression [7]. Although the function of ABCG2 was not tested, hESCs expressing HIF-2alpha, NANOG, and OCT4 correlated with decreased reactive oxygen species (ROS), increased glutathione (GSH), increased survival (as depicted by colony forming assays), and increased cytoprotection from intracellular insults [7]. Another recent report suggests that functional ABCG2 protects hESCs from physical stress such as UV irradiation [8]. Future studies should determine whether the role of ABCG2 in differentiation and cytoprotection is correlative or causative.

In summary, current studies suggest that ABCG2 expression is tightly regulated at both the transcriptional and translational levels. These dedicated regulations may play important roles in cytoprotection and differentiation of hESCs. Alterations in transcriptional and translational modifications, protein interactions, and signals from the microenvironment could all result in altered ABCG2 expression and function. Although ABCG2 is thought to have a myriad of roles, it is also functionally redundant with other $\mathrm{ABC}$ transporters. Therefore, a parallel analysis of ABCG2 expression with other $\mathrm{ABC}$ transporters in hESCs would enable us to understand their coordinated roles in human embryonic development, physiology, and pathology.

\section{Acknowledgments}

This work was supported by the Intramural Research Program of the National Institutes of Health (NIH) at the National Cancer Institute and the National Institute of Neurological Disorders and Stroke. We would like to thank Barbara Mallon, Pamela Robey, and Ronald McKay for their support of this project. We thank Misty Handley for preparing the figures and helpful discussion. We would also like to thank George Leiman for editorial assistance.

\section{Conflicts of Interest}

The authors declare no conflicts of interest.

\section{References}

1. Gottesman MM, Fojo T, Bates SE (2002) Multidrug resistance in cancer: role of ATP dependent transporters. Nat Rev Cancer 2(1): 48-58. [PubMed]

2. Robey RW, lerano C, Zhan Z, Bates SE (2011) The challenge of exploiting ABCG2 in the clinic. Curr Pharm Biotechnol 12(4): 595-608.[PubMed]

3. Dean M, Fojo T, Bates S (2005) Tumour stem cells and drug resistance. Nat Rev Cancer 5(4): 275-284.[PubMed]

4. Padmanabhan R, Chen KG, Gillet JP, Handley M, Mallon BS, et al. (2012) Regulation and expression of the ATP-binding cassette transporter ABCG2 in human embryonic stem cells. Stem Cells 30(10): 2175-2187.[PubMed]

5. Zeng H, Park JW, Guo M, Lin G, Crandall L, et al. (2009) Lack of ABCG2 expression and side population properties in human pluripotent stem cells. Stem Cells 27(10): 2435-2445.[PubMed]

6. Apáti A, Orbán TI, Varga N, Németh A, Schamberger A, et al. (2008) High leve functional expression of the ABCG2 multidrug transporter in undifferentiated human embryonic stem cells. Biochim Biophys Acta 1778(12): 2700-2709. [PubMed]

7. Das B, Bayat-Mokhtari R, Tsui M, Lotfi S, Tsuchida R, et al. (2012) HIF-2a suppresses $p 53$ to enhance the stemness and regenerative potential of human embryonic stem cells. Stem Cells 30(8): 1685-1695.[PubMed]

8. Erdei Z, Sarkadi B, Brózik A, Szebényi K, Várady G, et al. (2013) Dynamic ABCG2 expression in human embryonic stem cells provides the basis for stress response. EurBiophys J 42(2-3): 169-179.[PubMed]

9. Chen KG, Mallon BS, McKay RD, Robey PG. (2014)Human pluripotent stem cell culture: considerations for maintenance, expansion, and therapeutics. Cell Stem Cell 14(1): 13-26.[PubMed]

10. Chen KG, Mallon BS, Johnson KR, Hamilton RS, McKay RD, et al. (2014) Developmental insights from early mammalian embryos and core signaling pathways that influence human pluripotent cell growth and differentiation. Stem Cell Res 12(3): 610-621.[PubMed]

11. Nakanishi T, Ross DD (2012) Breast cancer resistance protein (BCRP $A B C G 2)$ : its role in multidrug resistance and regulation of its gene expression. Chin J Cancer 31(2): 73-99.[PubMed]

12. Chen KG, Szakács G, Annereau JP, Rouzaud F, Liang XJ, et al. (2005) Principal expression of two mRNA isoforms (ABCB 5alpha and ABCB 5beta) 
Citation: Padmanabhan R, Chen KG, Gottesman MM (2014) Lost in Translation: Regulation of ABCG2 Expression in Human Embryonic Stem Cells. J Stem Cell Res Ther 4: 180. doi:10.4172/2157-7633.1000180

of the ATP-binding cassette transporter gene ABCB5 in melanoma cells and melanocytes. Pigment Cell Res 18(2): 102-112.[PubMed]

13. Byrne JA, Strautnieks SS, Ihrke G, Pagani F, Knisely AS, et al. (2009) Missense mutations and single nucleotide polymorphisms in ABCB11 impair bile salt export pump processing and function or disrupt pre-messenger RNA splicing. Hepatology 49(2): 553-567.[PubMed]

14. To KK, Zhan Z, Litman T, Bates SE (2008) Regulation of ABCG2 expression at the 3' untranslated region of its mRNA through modulation of transcript stability and protein translation by a putative microRNA in the S1 colon cancer cell line. Mol Cell Biol 28(17): 5147-5161.[PubMed]

15. To KK, Robey RW, Knutsen T, Zhan Z, Ried T, et al. (2009) Escape from hsamiR-519c enables drug-resistant cells to maintain high expression of ABCG2. Mol Cancer Ther 8(10): 2959-2968.[PubMed]

16. Diop NK, Hrycyna CA (2005) N-Linked glycosylation of the human ABC transporter ABCG2 on asparagine 596 is not essential for expression, transport activity, or trafficking to the plasma membrane. Biochemistry 44(14): 5420 5429.[PubMed]

17. Xie Y, Xu K, Linn DE, Yang X, Guo Z, et al. (2008) The 44-kDa Pim-1 kinase phosphorylates BCRP/ABCG2 and thereby promotes its multimerization and drug resistant activity in human prostate cancer cells. J Biol Chem 283(6) 3349-3356.[PubMed]

18. Mogi M, Yang J, Lambert JF, Colvin GA, Shiojima I, et al. (2003) Akt signaling regulates side population cell phenotype via Bcrp1 translocation. J BiolChem 278(40): 39068-39075.[PubMed]

19. Bleau AM, Hambardzumyan D, Ozawa T, Fomchenko El, Huse JT, et al. (2009) PTEN/PI3K/Akt pathway regulates the side population phenotype and ABCG2 activity in glioma tumor stem-like cells. Cell Stem Cell 4(3): 226-235.[PubMed] 